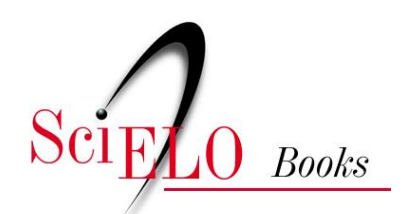

\title{
Experiencias de buenas prácticas \\ 11. Apoyos para garantizar el acceso al aprendizaje de los estudiantes con discapacidad en el nivel superior
}

\author{
Miriam Gallego \\ Mauricio Suárez \\ Andrés Jaramillo
}

\section{SciELO Books / SciELO Livros / SciELO Libros}

GALLEGOS, M., SUÁREZ, M., and JARAMILLO, A. Apoyos para garantizar el acceso al aprendizaje de los estudiantes con discapacidad en el nivel superior. In: GALLEGOS NAVAS, M., ed. La inclusión de las TIC en la educación de personas con discapacidad: relatos de experiencias [online]. Quito: Editorial Abya-Yala, 2019, pp. 155-163. ISBN: 978-9978-10-495-8. https://doi.org/10.7476/9789978104958.0014.

International license.

Todo o conteúdo deste trabalho, exceto quando houver ressalva, é publicado sob a licença Creative Commons Atribição 4.0.

Todo el contenido de esta obra, excepto donde se indique lo contrario, está bajo licencia de la licencia $\underline{\text { Creative }}$ Commons Reconocimento 4.0. 
155

Figura 79

Uso de información digital en su entrevista de trabajo

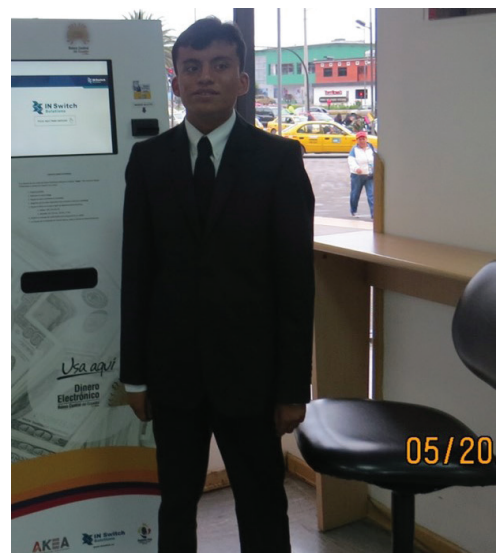

Foto: Silvania Salazar (2016)

\section{Apoyos para garantizar el acceso al aprendizaje de los estudiantes con discapacidad en el nivel superior}

"Los apoyos son recursos y estrategias cuyo objetivo es promover el desarrollo, la educación, los intereses y el bienestar personal de una persona y que mejoran el funcionamiento individual"

(Luckasson)

Universidad Politécnica Salesiana

Ecuador

Quito

Responsable: Mauricio Suárez y Andrés Jaramillo

Gestores de la práctica: Mara Tamayo Directora de la Biblioteca de la UPS

Garantizar la inclusión de estudiantes con discapacidad es un desafío para las instituciones de educación superior. La inclusión educativa tiene que enfocarse en la apropiación de aprendizajes que permitan a los estudiantes alcanzar el perfil de salida planteado para las diferentes carreras, de esta manera en la Universidad Politécnica Salesiana se cuenta con un espacio específico para la adaptación de recursos bibliográficos en formatos accesibles. 
156

\section{Motivación}

La Tiflobiblioteca de la Universidad Politécnica Salesiana, forma parte de la biblioteca de la Universidad, es un servicio creado hace diez años con el fin de apoyar académicamente a los estudiantes con discapacidad visual y a la comunidad en general cumple con la función de elaborar recursos bibliográficos accesibles.

Existe un alto número de estudiantes con discapacidad visual que al iniciar la etapa universitaria, no cuentan con la preparación adecuada para el uso del internet o que no tienen acceso a internet desde sus hogares situación que limita su acceso al conocimiento en igualdad de condiciones que el resto de los estudiantes.

\section{Objetivos}

- Facilitar la accesibilidad de los estudiantes con discapacidad visual a recursos didácticos accesibles.

- Capacitar a los estudiantes con discapacidad visual al uso de entonarnos virtuales y herramientas de comunicación y consulta.

\section{Desarrollo de la experiencia}

En concordancia con las necesidades y objetivos planteados, la atención a las necesidades de los estudiantes se da en dos etapas: accesibilidad a textos escritos y capacitación para el uso de las TIC.

\section{Accesibilidad a textos en tinta y digitales}

La tiflobiblioteca cuenta con un espacio físico equipado con once computadores y un escáner de alta resolución, dispositivos Víctor Reader producto de la gestión del grupo de investigación de Educación Inclusiva GEI. A este espacio asisten docentes, estudiantes y personas de la comunidad. 
157

Figura 80

Uso de la tiflobiblioteca

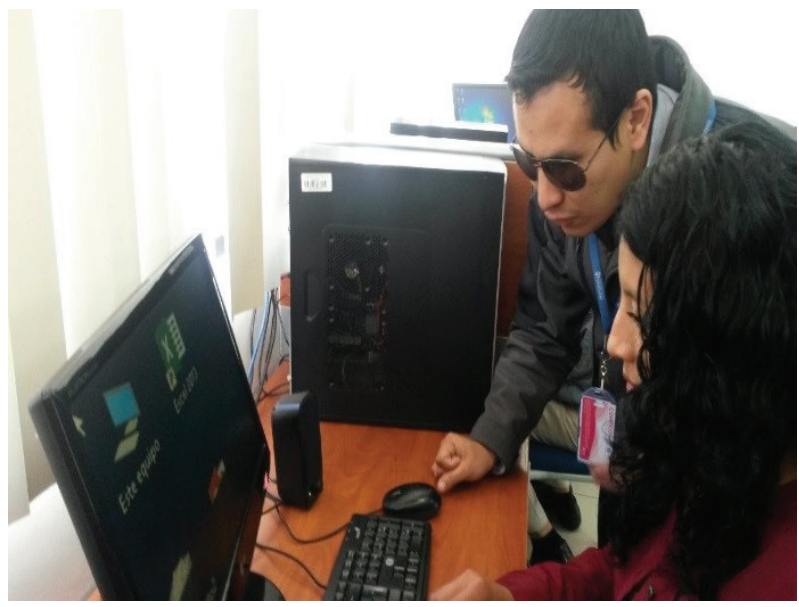

Foto: Miriam Gallego (2017)

La ruta a seguir por parte de los usuarios (docentes, estudiantes con discapacidad no solo de la universidad sino de la comunidad) para la elaboración de recursos bibliográficos accesibles se resume en los siguientes pasos:

- Solicita el servicio de digitalización del material según sus necesidades, generalmente los usuarios traen con sí, ejemplares originales, copias o archivos digitales que permitan ser transformados a imagen a texto. Para ello se cuenta con el apoyo de un escáner de alta resolución, con el cual se puede obtener un texto en formato Word, que luego, puede ser leído en la computadora o se lo transforma en audio para que pueda ser escuchado en algún reproductor de sonido.

- Búsqueda de información en el repositorio bibliográfico. La Tiflobiblioteca cuenta con 18000 títulos recopilados generados a partir ésta experiencia. 
158

- Si el material solicitado se encuentra en el repositorio bibliográfico se procede a ser entregado inmediatamente al usuario.

- Una vez concluido con el proceso de digitalización, y si el usuario necesita, se procede a la capacitación en el manejo del lector de pantalla JAWS, así como de los programas que vayan a emplear para cumplir con sus objetivos.

Es importante indicar que el uso de dispositivos móviles como el "Víctor Reader" permiten a la persona con discapacidad visual o cualquier persona que desee escuchar libros en diversos formatos, escuchar documentos de textos, música y libros en formato mp3; este dispositivo incluye una grabadora digital integrada para registrar notas de voz. En pocas palabras este recurso se convierte en una biblioteca móvil de gran apoyo para los estudiantes.

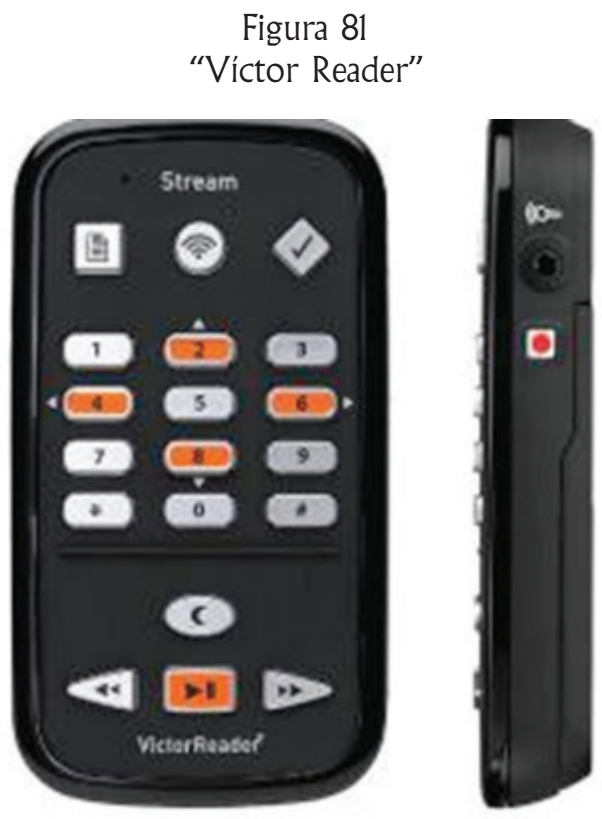

Fuente: Compartolid.es. Recuperado de https://goo.gl/gBNsjH 
159

Al igual que los lectores de pantalla el Víctor Reader es un dispositivo que permite la accesibilidad, autonomía e igualdad de oportunidades a los estudiantes con discapacidad visual.

\section{Capacitación para el uso de las TIC}

La capacitación a los estudiantes con discapacidad visual en el uso de las TIC se fundamenta en el conocimiento de lectores de pantalla como el programa Jaws y el NVDA.

La capacitación al uso del lector de pantalla Jaws se desarrolla bajo un ciclo de aprendizaje que conjuga la teoría con la práctica y permite el dominio de esta herramienta para acceso a la información y al cocimiento.

Figura 82

Ciclo de aprendizaje del programa Jaws

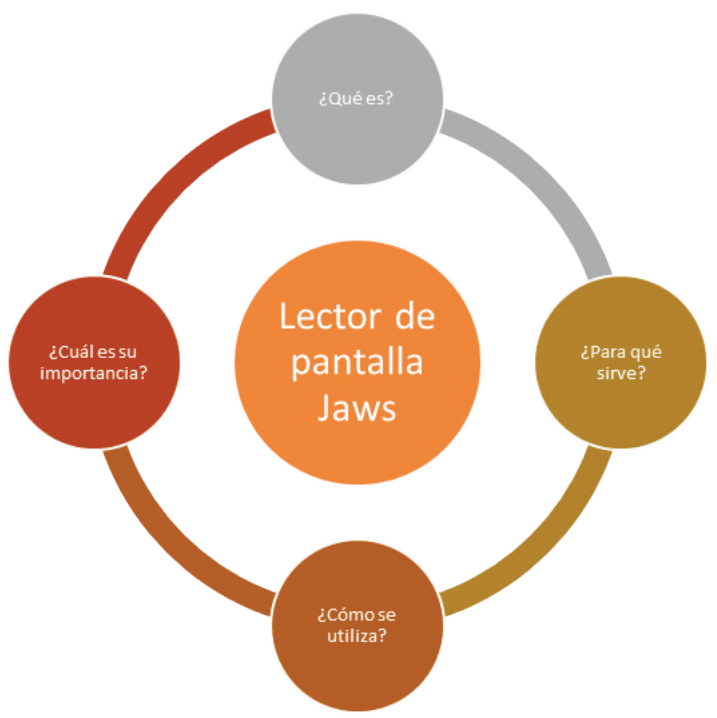

Fuente: Miriam Gallegos (2017) 
160

Figura 83

Uso del Software Jaws

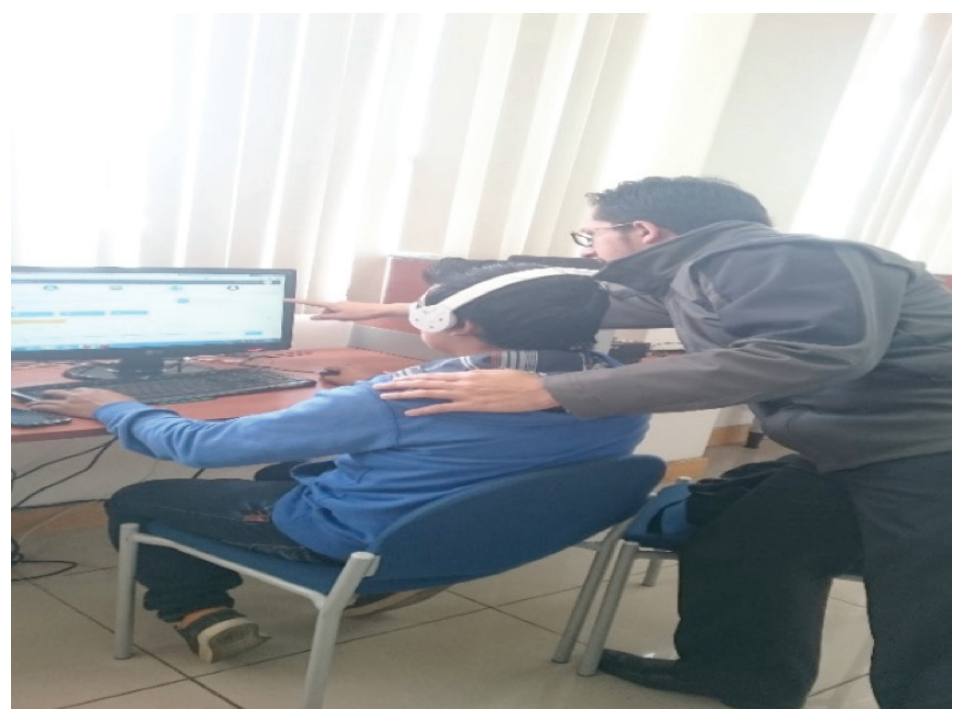

Foto: Andrés Jaramillo (2017)

Para conseguir este propósito, el programa convierte el contenido de la pantalla en sonido de manera que el estudiante pude acceder a todos las funciones del computador y navegar en internet. Para un eficiente manejo del programa Jaws, se requiere, que el modulador de voz sea regulado tanto en volumen como en velocidad de reproducción de acuerdo al manejo del mismo.

En el caso de estudiantes que se están iniciando en el uso del programa, fue necesaria una reproducción más pausada para que comprendan la acción que estaban realizando. Cuando el usuario adquiriere mayor experiencia en el manejo del computador mediante el Jaws, se aumenta el nivel de velocidad de reproducción de la voz y las acciones y comandos que se estén ejecutando en el computador se realizan con mayor rapidez. 


\section{1}

\section{Figura 84}

Lectores de pantalla

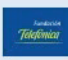

\section{Lectores de pantalla}

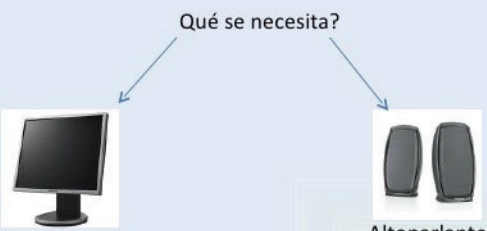

Computadora

(puede usarse el monitor apagado)

Altoparlantes

QQ Usuarios: personas ciegas, personas con Déficit cognitivo o personas con graves

Trastornos visuales.

Nos brindan la información auditivamente

Fuente: Lectores de Pantalla y Accesibilidad Web. Recuperado de https://goo.gl/fMWa1V

\section{Figura 85}

Usuarios de la Tiflobiblioteca

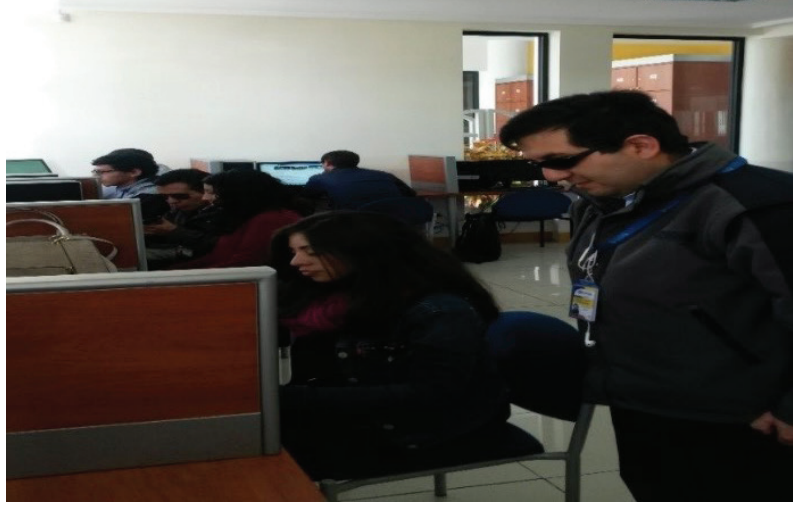

Foto: Miriam Gallego (2017) 
A los usuarios de la Tiflobiblioteca se los capacita en el manejo de correos electrónicos con mayor compatibilidad con Jaws. Se les enseña el manejo del Outlook (Personal e institucional) y Gmail. El servidor Gmail es el más accesible para las personas con discapacidad visual, en él se puede modificar la vista estándar a una vista básica, aunque, por un lado pierde características como plug-ins, imágenes y acceso ilimitado a videos, por otro lado permite resaltar el fácil acceso y manejo de la información textual.

Una herramienta importante para el aprendizaje universitario en los estudiantes con discapacidad visual son los dispositivos móviles (Smartphone). Los estudiantes reciben una clase introductoria para el uso de lectores de pantalla para estos dispositivos. En algunos casos se instalan moduladores de voz para dispositivos móviles, como Google Talkback para Android o VoiceOver para IOS.

Las actividades del proceso de formación son eminentemente prácticas entre las más relevantes constan las siguientes:

Figura 86

Actividades del proceso de formación

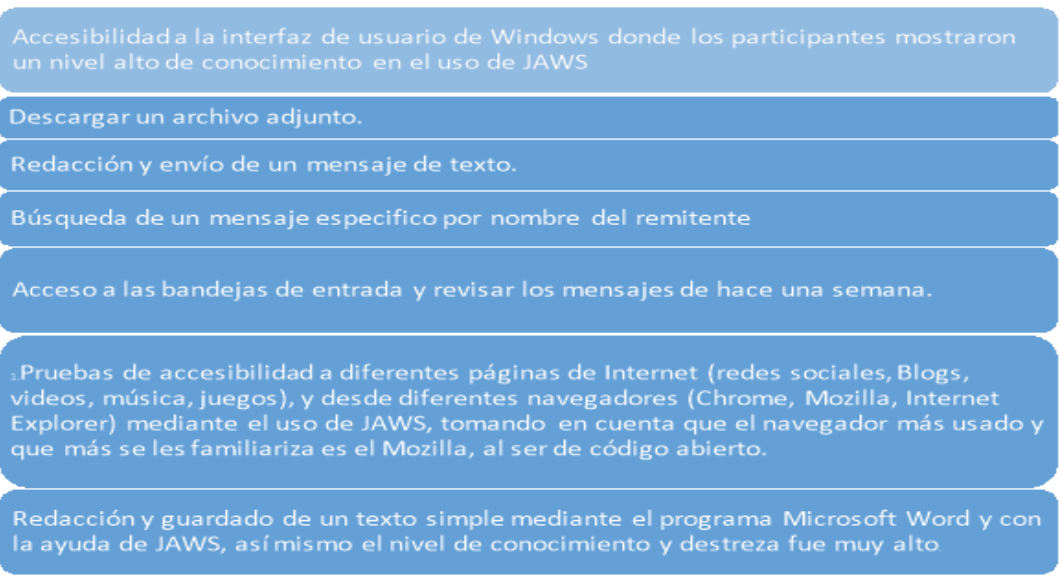

Fuente: Miriam Gallego (2017) 


\section{3}

\section{Logros obtenidos}

Dentro de los resultados alcanzados se presentan los estudiantes:

- Mayor accesibilidad a la interfaz del correo electrónico al usar la versión básica que Gmail proporciona a los usuarios. Los estudiantes demuestran mayor acceso a los mensajes recibidos.

- Capacidad para encontrar un documento y en algunos casos a modificar el nombre y a adjuntarlo al correo electrónico.

- Acceso a información y a textos escritos tanto académicos como de interés personal con mayor rapidez.

- Facilidad de comunicación e interacción con la información de la universidad.

- Mayor de independencia en las actividades académicas.

Con los conocimientos adquiridos se procede a elaborar una lista de cotejo con las actividades antes descritas como instrumento de evaluación para medir los logros obtenidos en los participantes, con resultados similares en cuanto a la práctica desarrollada.

Es importante resaltar que el aprendizaje es evaluado mediante la práctica constante del uso de las herramientas, los encargados de la evaluación los encargados de la Tiflobiblioteca quienes desempeñan el rol de tutores.

\section{Recursos utilizados}

- Computadores del departamento de Tiflobiblioteca.

- Programa JAWS v.17: Es un software lector de pantalla para ciegos o personas con visión reducida.

- Internet (redes sociales, correo electrónico, blogs, videos, música): es un conjunto descentralizado de redes de comunicación interconectadas entre sí.

- Dispositivos Víctor Reader. 